\title{
Segmentation of Lung Tumor in CT Scan Images using FA-FCM Algorithms
}

\author{
P.Kalavathi ${ }^{1}$ and A.Dhavapandiammal ${ }^{2}$ \\ ${ }^{1}$ Gandhigram Rural Institute - Deemed University, Tamil Nadu) \\ ${ }^{2}$ M.Phil Research Scholar, Madurai Kamaraj University (Evening College), Tamil Nadu)
}

\begin{abstract}
Lung Cancer is dangerous disease that cause most human to death at early age and it is an uncontrolled cell growth in tissues on the lung. Many algorithms and technologies are introduced for identifying the lung tumors and it does not provide efficient accuracy. Our proposed system introduces a new identification process for Lung tumor. Here, we introduced a novel algorithm named FA-FCM which is a combination of firefly algorithm and Fuzzy C-means clustering. The segmented output image is compared with expert segmented image in terms of Jaccard and Dice similarity values. Our proposed method produced better result on all the tested images compared with the gold/expert segmented images.
\end{abstract}

Keywords: Fuzzy C-means algorithm, Firefly algorithm, Lung Tumors, Segmentation

\section{Introduction}

Biomedical image processing is a very broad field; it covers biomedical signal gathering, image forming, picture processing, and image display for medical diagnosis based on features extracted from images. Recently, image processing techniques are widely used in medical areas for image improvement in order for earlier detection and treatment of several pathologies. Lung cancer seems to be the common cause of death among people throughout the world. Early detection of lung cancer can increase the chance of survival among people. Lung cancer is a malignant lung tumor characterized by uncontrolled cell growth in tissues of the lung. If it is left untreated, this growth can spread beyond the lung by the process of metastasis into nearby tissue or other parts of the body. Most cancers that start in the lung, known as primary lung cancers, are carcinomas. The two main types are small-cell lung carcinoma (SCLC) and non-small-cell lung carcinoma (NSCLC). The most common symptoms are cough, weight loss, shortness of breath and chest pains.

The 85 percent of lung cancer arise are due to long-term tobacco smoking. About 10-15 percent of cases occur due to genetic factors and exposure to radon gas, asbestos, second-hand smoke, or other forms of air pollution. Lung cancer may be seen on chest radiographs and computed tomography (CT) scans. The diagnosis is confirmed by biopsy which is usually performed by bronchoscopy or CT-guidance. Treatment and long-term outcomes depend on the type of cancer, the degree of spread, and the person's overall health. Common treatments include surgery, chemotherapy and radiotherapy. NSCLC is sometimes treated with surgery, whereas SCLC usually responds better to chemotherapy and radiotherapy. CT scanned lung images of cancer patients are acquired from various hospitals. Using image processing techniques like pre-processing and feature extraction area of interest is separated.

Segmentation is an essential process for medical image processing and it is used to segment and analyze the pathologies present in the medical images for accurate and fast diagnosis and better treatment. Many segmentation methods have been developed for medical images especially for brain images [1]-[6].

Lung segmentation is mainly classified into five major methods such as Thresholding based methods, Region based methods, Shape based methods, Neighboring anatomy-guided methods and Machined learning based methods [7]. The thresholding based method fails to deal with the attenuation variations and then it fails to categorize pathologic classifications. Whereas, the region based segmentation, will not able to segment the regions with moderate to higher level of abnormalities. The neighboring anatomy-guided methods and machined learning based methods are efficient but are computationally expensive.

Marker-controlled watershed segmentation method introduced in [10] involves with the computation of segmentation function, foreground markers, background markers and finally compute watershed transform of the modified segmentation function. This segmentation method is little complex and the accuracy is not upto the expected level.

Lung tumor segmentation proposed in [11] uses level set model with modified morphological gradient. According to the physiological characteristics of lung cancer, the tumor is generally located in the lung, and some special adhering tumors can be connected to the lung area. In CT images, the texture, gray scales and shape features of lung cancer are obviously different from those of other tissues, therefore their statistical characteristics are used to distinguish the tumor and background of an image. 
In [12], the graph-cut based segmentation algorithm was used to segment the lung tumor. This method takes up the weighted graph and computes the edges to cut and gives accurate results only for minimum number of CT images. Respiratory system segmentation [13] was used for the process of removing the trachea and primary bronchi present in the CT lung image. Itai et al [14] developed a method using snake segmentation method. Snake is one of the most popular active contour models which defines an initial curve within the image and then it grow up to reach the object boundary under the influences of the internal and external forces computed based on image statistics information.

Bandyopadhyay et al [15] designed a method for lung boundary detection which deals with anatomical segmentation, which segments the lung by using its region of interest. This anatomical segmentation fails to spot some of the significant structures present in the CT image. Active contour based image segmentation was devised in [16] uses Euclidean curve shortening flow to split the image into several regions. Then the gradient of this segmentation map gives the region constraints in the vicinity of the region boundary map.

A method proposed in [17] uses discontinuity and similarity in the intensity of a lunch image as two important properties to segment the lung cancer in the CT lung image. Thresholding method is a significant method for the purpose of segmenting a medical mage for the purpose of identification of tumors [18], [19]. Thresholding is broadly classified into two categories, one is global thresholding and the other is local thresholding. This thresholding method removes the background by using mean and the standard deviation. The global thresholding method is further sub-classified into three such as Traditional (Otsu's), Iterative (Triclass) and Multistage (QIR) [20]. Thresholding by histogram approach takes less storage space compared to other methods but it gives lesser accuracy when compared with watershed segmentation approach. Mesanovic et al [21] proposed a segmentation technique which is based on histogram thresholding and region growing algorithm. In this approach it is necessary to specify the seed points to get better result [22].

In this paper, an effective hybrid method for accurate segmentation of lung tumor in CT scan image is presented. This method uses novel algorithms to detect the tumor more accurately and to reduce the detecting time. The remaining part of the paper is organized as follows: In section 2 , the methodological detail of the proposed method is given, the results and discussion are given in section 3 and the conclusion is given in section 4.

\section{Methodology}

The overall block diagram of the proposed method is given in Fig 1.In this method, an effective hybrid method for segmenting tumor in the lung CT scan image is proposed. This method uses fire-fly (FA), Fuzzy Cmeans (FCM) and region growing algorithms to detect lung tumor. This method first read the input image of Lung tumor which is in gray scale, and the noise is removed by median filter. Then we use the firefly algorithm for getting optimized clusters (centroids) for getting alarm pixels from our preprocessed image. The identified alarm pixels are forwarded to region growing and finally it is followed by fuzzy c-means for segmentation. Segmentation is done based on fuzzy index, this displays the segmented results. Then the output of segmentation is compared with the gold standard image for the performance analysis by computing Jaccard similarity and Dice coefficient values.

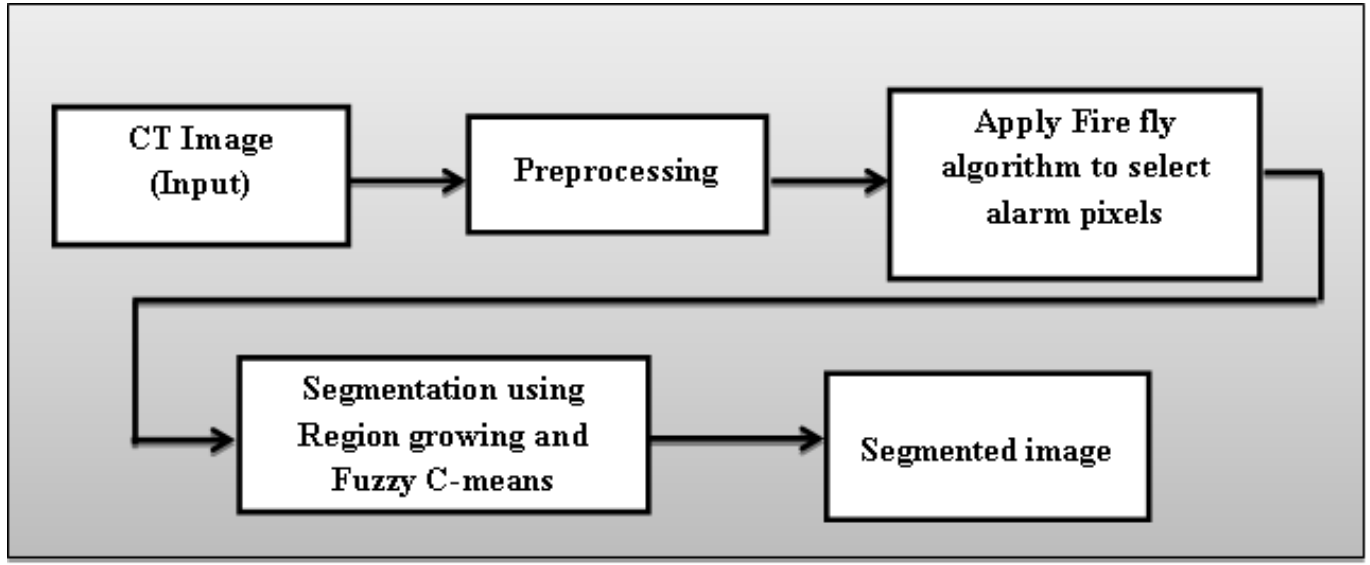

Figure 1: Block diagram for the proposed method

\subsection{Algorithm 1 (FA)}

Firefly algorithm (FA) is employed for the purpose of improving the segmentation method, for the purpose of effective prediction of the presence of lung tumors. This firefly algorithm is established with three ideal rules as given below:

- $\quad$ All the fireflies are equal and hence they can attract all the other fireflies. 
- Attractiveness is proportional to their brightness, thus for any two flashing fireflies, the less bright one will move towards the brighter one. The attractiveness is proportional to the brightness and they both decrease as their distance increases. If there is no brighter one than a particular firefly, it will move randomly.

- Brightness of a firefly is affected or determined by the landscape of the objective function.

The brightness of the fireflies decreases with the distance from the light source, therefore, the attractiveness of a firefly function determined by the following monotonically decreasing function. The attractiveness of the firefly is directly proportional to the value of light intensity by adjacent, hence the equation is given as,

$$
\begin{aligned}
\beta & =\beta_{0} e^{-\gamma r 2} \\
r_{i, j} & =\sqrt{\left(x_{i}-x_{j}\right)^{2}+\left(y_{i}-y_{j}\right)^{2}}
\end{aligned}
$$

Where, ' $r$ ' is the distance between each two fireflies and $\beta_{0}$ is their attractiveness at $r=0, \gamma$ is the absorption coefficient. Then the distance between any two fireflies $\mathrm{i}$ and $\mathrm{j}$ at $\mathrm{x}_{\mathrm{i}}$ and $\mathrm{x}_{\mathrm{j}} \mathrm{can}$ be Cartesian distance, which is given as $r_{i, j}$.

The graphical representation of centroid calculation using the Firefly algorithm is shown in the Fig 2.

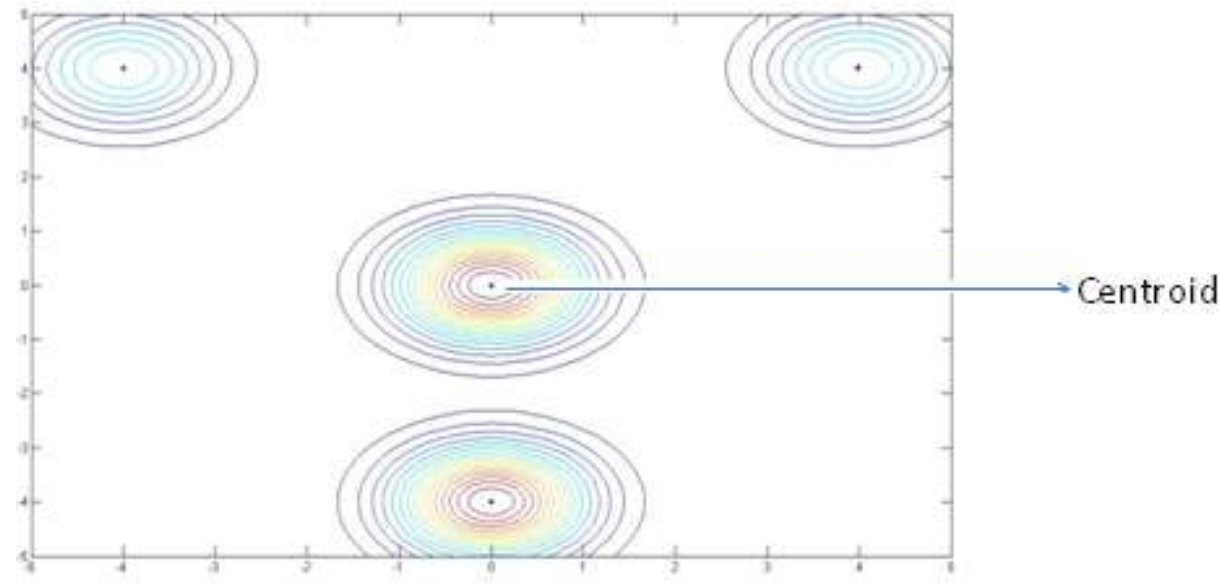

Figure 2: Centroid computation using Firefly algorithm

\subsection{Algorithm 2 (FCM)}

Fuzzy C-means algorithm is developed by modifying the objective function of the standard FCM algorithm with a penalty term that takes into account the influence of the neighboring pixels on the center pixels. Fuzzy segmentation has been favored over hard segmentation in some medical image applications. Since partial volume effects and image noise reduces the accuracy of hard segmentation. To cope with the limits of hard segmentation, a fuzzy c-means clustering method is employed to classify pixels into several tissue categories. The algorithm is an iterative clustering method that produces an optimal ' $c$ ' partition by minimizing the weighted within the group sum of squared error objective function $\mathrm{J}_{\mathrm{FCM}}$.

$$
J_{F C M}=\sum_{c=1}^{C} \sum_{j=1}^{J} u_{j c}^{2}\left\|I_{j}-v_{c}\right\|^{2}
$$

where, $v_{c}$ the centroid of class ' $c$ ' and ' $C$ ' is the total number of tissue classes $u_{j c}$, is the membership value of pixel ' $j$ ' for class ' $c$ ' and requires $u_{j c} \in[0,1]$ subject to $\sum_{c=1}^{C} u_{j c}=1, ' J$ ' is the total number pixels in the image and ' $I_{j}$ ' is the image intensity at the position ' $j$ '.

The objective function is minimized when a large membership is assigned to pixel close to the centroid of a class and a small membership value is assigned to a pixel far away from the centroid. This is a nonlinear problem and it can be solved iteratively. During each iteration, a new set of membership functions and class centroids are computed based on the above Equation(3). The steps involved in FCM are given below:

Step 1 - The initial values for the class of centroids is represented as $v_{c}, c=1, \ldots \ldots \ldots . C$

Step 2 - The membership values are calculated as per the following formula:

$$
u_{j c}=\frac{\left\|I_{j}-v_{c}\right\|^{-2}}{\sum_{i=1}^{C}\left\|I_{j}-v_{c}\right\|^{-2}}
$$


Step 3 - Calculate the new centroid using the following equation.

$$
v_{c}=\frac{\sum_{j=1}^{J} \boldsymbol{u}_{j c}^{2} \boldsymbol{I}_{j}}{\sum_{j=1}^{J} \boldsymbol{u}_{j c}^{2}}
$$

Step 4 -Repeat Step-2 and Step-3 until the algorithm reaches convergence. Convergence will be reached when there is a maximum change in the computed centroids between the previous two iterations.

The process of lung tumor segmentation is given in Fig 3.

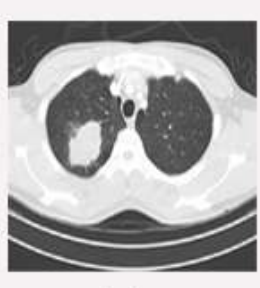

(a)

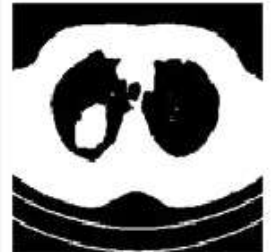

(b)

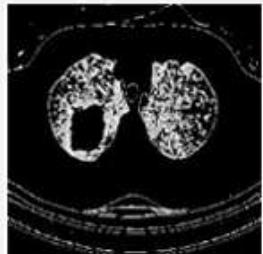

(c)

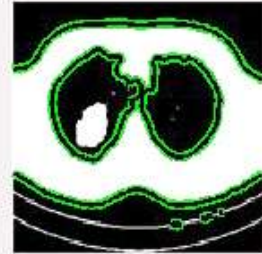

(d)

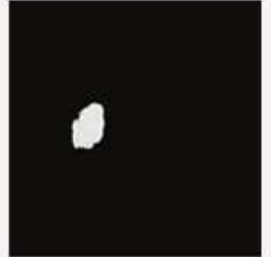

(e)

Figure 3: Process of lung tumor segmentation (a) original image (b) preprocessed image (c) output of firefly segmentation (d) segmentation using region growing and FCM (e) segmented lung tumor

In Figure 3, the original input image is shown in Figure 3(a), the preprocessed image is shown in Figure 3(b), the output for the firefly segmentation method is shown in Figure 3(c), the output is given to region growing method and forwarded for the FCM method and the resultant image is shown in Figure 3(d) and the final segmented image is shown in Figure 3(e).

The summary of the steps involved in the proposed method is given below:

Step 1: Read the original image.

Step 2: Remove the noise in the given input image by median filter(preprocessing).

Step 3: Define the centroid points.

Step 4: Obtain the segmented image by using the computed centroid at Step 3.

Step 5: Obtain the final segmented tumor image.

Step 6: Compute the similarity measure using the segmented image by the proposed and Expert segmentation image.

\subsection{Performance Evaluation}

In our proposed method the performance is evaluated using the similarity measures Jaccard (J) and Dice (D)[6].

The Jaccard $(\mathrm{J})$ value is calculated by:

$$
J(S 1, S 2)=\frac{|S 1 \cap S 2|}{|S 1 \cup S 2|}
$$

The Dice (D) value is calculated by:

$$
D(S 1, S 2)=2 \frac{|S 1 \cap S 2|}{|S 1|+|S 2|}
$$

Where,S1 is the segmented image and $\mathbf{S 2}$ is the original image (ground truth image).

\subsection{Datasets Used}

To evaluate the performance of the proposed method, we use the lung CT images from the dataset [25]. The Vision and Image Analysis group/International Early Lung Cancer Action Program (VIA/I-ELCAP) is a public access research database. The database consists of set of 50 whole-lungs CT images with tumor. It was created to make available a common dataset that may be used for the performance evaluation of lung cancer detection techniques. 


\section{Results and Discussion}

In our proposed method, we have used sample CT images from the dataset [25] for evaluating the performance of our proposed method. The quantitative evaluation is done by computing the values of Jaccard (J) and Dice (D) similarity measures. In Figure 4, the sample CT lung images from the dataset are shown along with their segmentation result by the proposed and expert method. It is evident from the Figure 4 and Table -1 is that this proposed method is efficient and effective to segment the lung tumor. The original lung CT image is given in column (a), the segmented tumor image by the proposed method is shown in Figure 4(b), and its corresponding expert segmented image is shown in Figure 4(c).The following table (Table 1)gives the computed Jaccard and Dice values for the images shown in Figure 4.

Table - 1 Computation of Jaccard (J) and Dice (D) similarity measures for the image shown in Fig 4.

\begin{tabular}{|l|l|l|l|}
\hline SI. No. & Images & Jaccard values(J) & Dice Values(D) \\
\hline 1 & Image 1 & 0.8705 & 0.9625 \\
\hline 2 & Image 2 & 0.8628 & 0.9131 \\
\hline 3 & Image 3 & 0.8511 & 0.9329 \\
\hline 4 & Image 4 & 0.8848 & 0.9885 \\
\hline 5 & Image 5 & 0.8772 & 0.9359 \\
\hline 6 & Image 6 & 0.8928 & 0.9324 \\
\hline 7 & Image 7 & 0.8601 & 0.9565 \\
\hline 8 & Image 8 & 0.8752 & 0.9432 \\
\hline 9 & Image 9 & 0.8619 & 0.9286 \\
\hline 10 & Image 10 & 0.8524 & 0.9527 \\
\hline
\end{tabular}

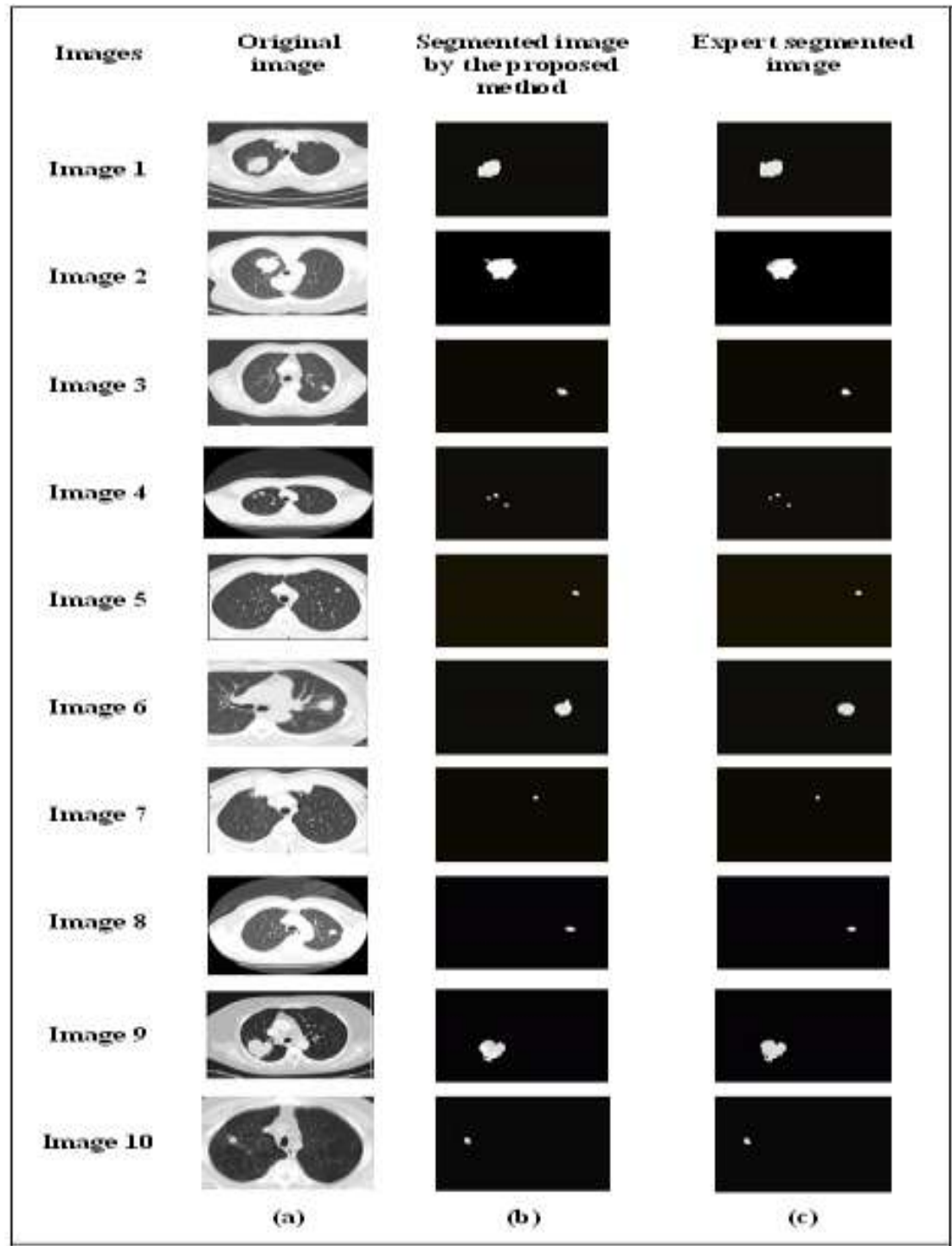

Figure 4: Segmentation result by the proposed method (a) Original images (b) Segmented tumor image by the proposed method (c) Expert segmented tumor image 


\section{Conclusions}

This proposed method describes a simple method for the segmentation and identification of lung tumor in CT scan images. In this method, we useda combined algorithm, Firefly Algorithm with Fuzzy C-Means for segmentation. This algorithm helps to identify the centroid points from an input image first by that they form a cluster to reduce the time on tumor segmentation. The computed Jaccard (J) and Dice (D) similarity measures using the expert segmented image produced better result on all the tested images.

\section{References}

[1]. P. Kalavathi and V.B. Surya Prasath, Method on skull stripping of MRI Head Scan Images-a Review, Journal of Digital Imaging, Springer, , Vol. 29, No. 3, pp. 365-379, 2016.

[2]. K. Somasundaram and P.Kalavathi, Brain Segmentation in magnetic Resonance Human Head Scan using multi-seeded Region Growing, Imaging Science Journal, Vol 62, no.5, pp. 273-284, 2014.

[3]. K. Somasundaram and P. Kalavathi, Contour-based brain segmentation method for magnetic Resonance Imaging Human Head Scans, Journal of Computer Assisted Tomography, vol.37, no.3, pp.353-368, 2013.

[4]. K.Somasundaram and P.Kalavathi, A Noval Skull Stripping Technique for T1-weighted MRI Human Head Scans, Acm digital library, pp.1-8, 2012.

[5]. K.Somasundaram and P.Kalavathi, Skull Stripping of MRI Head Scans Based on chan-vese Active Contour model, International Journal of Knowledge Management \& e-learning, vol.3, No.1, pp.7-14, 2011.

[6]. K.Somasundaram and P.Kalavathi, A Hybrid Method for Automatic Skull Stripping of magnetic Resonance Images (MRI) of Human Head Scans, IEEE Xplore digital library, pp.1-5, 2010.

[7]. A. Karthikeyan, M. Valliammai, "Lungs Segmentation using Multi-level Thresholding in CT Images", International Journal of Electronics and Computer Science Engineering, 2012.

[8]. E.E. Mohd. A Muhd. M.M. Hatta, Z. Htike and S.L. Win, "Tumor Detection in Medical Imaging: A Survey”, International Journal of Advanced Information Technology (IJAIT) Vol. 4, No. 1, 2014.

[9]. A. Mansoor, U. Bagci, B. Foster, Z. Xu, G. Z. Papadakis, L.R. Folio, J. K. Udupa, D. J. Mollura, "Segmentation and Image Analysis of Abnormal Lungs at CT: Current Approaches, Challenges, and Future Trends", Radio Graphics Informatics, 2015.

[10]. V.A.Gajdhane, Deshpande, "Detection of Lung Cancer Stages on CT Scan Images by Using Various Image Processing Techniques", IOSR Journal of Computer Engineering, Volume 16, Issue 5,2014.

[11]. W.X Peng, Z. Wen, C. Ying, "Tumor segmentation in lung CT images based on support vector machine and improved level set", Open Electronics Letters, Vol 11, No 5, 2015.

[12]. R.Pinho, V.Delmon, J. Vandemeulebroucke, S. Rit1, and D. Sarrut, Keuhkot “A Method for Lung Segmentation”, Research Gate, 2011.

[13]. Y.D. Cid, O.A Alfonso, J.D. Toro, A. Depeursinge, H. Muller, "Efficient and fully automatic segmentation of the lungs in CT volumes", IEEE International Symposium on Biomedical Imaging, 2015.

[14]. Y Itai, H Kim, S Ishikawa, A Yamamoto and K Nakamura, "A Segmentation Method of Lung Areas by Using Snakes and Automatic Detection of Abnormal Shadow on the Areas", International Journal of Innovative Computing, Information and Control Volume 3, Number 2, 2007.

[15]. S.K.Bandyopadhyay, "A Method for Lung Boundary Detection", International Journal of Information and Communication Technology Research, Volume 2 No. 2, 2012.

[16]. A. Osareh, and B.Shadgar, "Automatic Segmentation of Lung Areas in Magnetic Resonance Images", World Academy of Science, Engineering and Technology International Journal of Computer, Electrical, Automation, Control and Information Engineering Vol 3, No 8, 2009.

[17]. S.Mokhled S. A.Tarawneh, "Lung Cancer Detection Using Image Processing Techniques", Leonardo Electronic Journal of Practices and Technologies, Issue 20, 2012.

[18]. N. Mesanovic,M. Grgic , H. Huseinagic, M.Males, E.Skejic , M.Smajlovic, "Automatic CT Image Segmentation of the Lungs with Region Growing Algorithm", Conference of Systems, 2011.

[19]. V. Parameshwarappa, Nandish, "Segmentation of Lung Cancer using Image Enhancement Techniques and Region Growing Algorithm”, International Journal of Engineering Research \& Technology (IJERT) Vol. 3 Issue 2, 2014.

[20]. Farah H. A. Jabar, waidah Ismail ,Rosalina A.Salam, Rosline Hassan, "Image Segmentation using a Hybrid Clustering Technique and Mean Shift for Automated Detection Acute Leukaemia Blood Cells Images", Journal of Theoretical and Applied Information Technology, Vol 76, No 1, 2015.

[21]. S. L. A. Lee, A. Z. Kouzani, and E. J. Hu, "Automated detection of lung nodules in computed tomography images: a review," Machine vision and applications, vol. 23, no. 1, pp. 151-163, 2012.

[22]. A. Farag, A. Ali, J. Graham, and et al., "Evaluation of geometric feature descriptors for detection and classification of lung nodules in low dose ct scans of the chest," in Proc. ISBI, 2011, pp. 169-172.

[23]. S. Diciotti, G. Picozzi, M. Falchini, and et al., "3-d segmentation algorithm of small lung nodules in spiral ct images," IEEE Trans. Information Technology in Biomedicine, vol. 12, no. 1, pp. 7-19, 2008

[24]. Y. Song, W. Cai, J. Kim, and D. Feng, "A multi-stage discriminative model for tumor and lymph node detection in thoracic images,” IEEE Trans. Medical Imaging, vol. 31, no. 5, pp. 1061-1075, 2012.

[25]. VIA/I-ELCAP Public Access Research Database. http://imaging.cancer.gov/programsandresources/InformationSystems/LIDC. 